\title{
Las colonias españolas en África durante el primer franquismo (1939-1959). Algunas reflexiones
}

\author{
Sergio Suárez Blanco*
}

RESUMEN

La revalorización de las "colonias" españolas en Átrica (Protectorado de

Marruecos, Ifni, Sahara, Guinea

Ecuatorial) durante el régimen de

Franco vino de la mano de las reivindicaciones territoriales esgrimidas en el transcurso de la Segunda Guerra Mundial con la pretensión de construir un Imperio colonial. Tras los excesos retóricos, la política africana fue impulsada por la Presidencia del Gobierno (Carrero) a través de la Dirección General de Marruecos y Colonias y del Instituto de Estudios Africanos. Esto dio pie a la constitución de un discurso africanista, que no desdeñó los aspectos económicos.

La preocupación del Estado, canalizada a través del Instituto Nacional de Industria (INI), para poner en explotación dichos territorios

\section{ABSTRACT}

The regime of General Franco amied at revaluating its colonies in Africa (Morocco's Protectorate, Ifni, Sahara, Equatorial Guinea) to form a Colonial Empire through territorial expansion during Worl War II. African policy was designed by Carrero Blanco, Deputy Minister to the Head of the Government through the Department of Morocco and Colonial Affairs and the Institute of African Studies. It led to an Africanist discourse that emphasized the economic aspects of the affair.

The desires to exploiting these African territories emanated from the general concern about fostering the industrilization of the country through an expansion of the home market and the search for new sources of raw materials. All these policies were undertaken by the State holding INI in

* Becario del Departamento de Historia e Instituciones Económicas II. Universidad Complutense de Madrid. 
respondia a la necesidad de complementar la economía metropolitana en los años de la autarquía (1939-59) mediante nuevos mercados y fuentes de materias primas.

Futuras investigaciones deben tanto especificar la explotación de cada territorio como encuadrarla en un marco comparativo, especialmente con

las colonias portuguesas e italianas.

\section{PALABRAS CLAVE Marruecos, Sahara, Guinea, franquismo, INI, colonialismo, autarquía, Carrero}

the selfsufficiency period between 1939 and 1959.

Future research will show how each territory was exploited. Furthermore there is a case to draw a comparision between Spanish, Portuguese and Italian colonial policies.

\section{KEY WORDS}

Morocco, Sahara, Equatorial Guinea, Franco's regime, INI, Colonialism, economic self-sufficiency, Carrero

Han pasado ya más de diez años desde que el profesor Víctor Morales Lezcano, uno de nuestros más reputados expertos en la presencia española en África y especialmente en Marruecos, afirmara que las colonias de África fueron para el franquismo vitrina de exhibición, factor de prestigio y fuente de beneficios para determinados sectores adictos a su estado de cosas ${ }^{1}$. Desde entonces muy poco se ha avanzado a la hora de emitir un juicio, siquiera sea provisional, acerca del papel que desempeñaron los territorios españoles en África (Protectorado de Marruecos, Sahara, Ifni, Guinea Ecuatorial) en la política (exterior, económica, militar, cultural...) del régimen de Franco.

Tal carencia resulta extraordinariamente llamativa si tenemos en cuenta que el golpe de Estado que dio origen a la Guerra Civil y al régimen de Franco tuvo su inicio en Marruecos y su más importante valedor en la rama africanista del Ejército y que, además, las postrimerías del régimen contemplaron a su vez el fin de la presencia española en África con la firma, en noviembre de 1975, de los acuerdos de Madrid por los que el Gobierno español entregaba la administración del Sahara a Marruecos y Mauritania. Y no podemos olvidar, por otra parte, la trascendencia que tuvo para la formación política, militar e, incluso, personal del general Franco su estancia en Marruecos. Hasta tal punto esto es así que el propio Franco le diría al periodista Manuel Aznar en una entrevista el 1 de enero de 1939: Sin África, yo apenas puedo explicarme a mi mismo?

Morales Lezcano, Victor (1986), p. 84

Cit. por Fusı, Juan Pablo (1985), p. 39. 
Por todo ello, es cada vez más necesaria una interpretación de conjunto sobre lo que realmente supuso la vertiente colonial o africana del régimen de Franco. Mientras tanto, tendremos que conformarnos con aproximaciones sectoriales y con la hipótesis de partida (las colonias africanas como fuente de prestigio y de beneficios) esbozada por Morales Lezcano. Como es lógi$\mathrm{co}$, el objetivo de estas páginas no es el de rellenar esa laguna en el conocimiento de nuestro pasado más reciente sino, de forma mucho más modesta, el de reflexionar en voz alta sobre algunos aspectos de la política africana del primer Franquismo y, en especial, acerca de la significación económica de los territorios africanos en el marco de la autarquía ${ }^{3}$.

Antes de seguir adelante conviene aclarar que aquí hago un uso lato sensu del término primer Franquismo prolongándolo desde 1939 a 1958/59, en contra del que hacen los historiadores de la política (hasta 1945) o de la economía (hasta 1951), por considerar que en el caso de las colonias africanas el cambio de rumbo no se produce hasta finales del decenio de los cincuenta con la independencia de Marruecos (abril de 1956) y la aplicación de la política de provincialización en Sahara e lf́ni (enero 1958) y Guinea Ecuatorial (julio 1959).

Se ha convertido ya en un tópico afirmar que España ha carecido de una política coherente y con unos objetivos claros durante el tiempo que tuvo una presencia activa en África (esto es, los siglos $x \mid x$ y $x x$ ). A grandes rasgos, da la impresión de haber sido una política errática, falta de continuidad y en exceso dependiente de la coyuntura tanto española como europea. Quizá la razón de ello deba buscarse en el carácter subalterno que tuvieron las colonias africanas en la política española, un papel que sólo abandonaron en las dos primeras décadas del xx por la repercusión de las campañas de Marruecos en la opinión pública y en el propio desarrollo de la vida política de la metrópoli. Si exceptuamos estos momentos de protagonismo debido a razones bélicas, no parece que la colonización de nuestras posesiones africanas fuera nunca un objetivo prioritario.

Por lo que se refiere al periodo que nos ocupa (1939-59) existen razones para pensar que tal situación habría sufrido un cambio cualitativo. En primer lugar, el poder ( $y$, en estos años, todo el poder y ejercido por lo demás de una forma exclusiva y autoritaria) se encuentra en manos de un

3 Estas reflexiones se enmarcan dentro de una investigación de mayor calado como es la de mi Tesis Doctoral en curso en torno al uso económico de las colonias africanas entre 1939 y 1959 y sus implicaciones en la política autárquica del régimen de Franco, en la que estoy trabajando bajo la dirección de Antonio Gómez Mendoza en el marco del Departamento de Historia e Instituciones Económicas II de la Facultad de CC. Económicas y Empresariales de la Universidad Complutense de Madrid. 
hombre, Franco, para el que África o, por mejor decir, Marruecos es una preocupación de primer orden. Junto a él, en los aledaños de poder, se sitúa una nutrida legión de generales cuya carrera se ha forjado en el Protectorado (Orgaz, Varela, Arganda, Jordana, Vigón, Kindelán...). Asi pues, el Ejército español en África se encuentra en la mejor situación para imponer sus intereses y puntos de vista.

En otro orden de cosas, la situación internacional contribuye a revalorizar la posesión de territorios en África. La Segunda Guerra Mundial es la gran ocasión que tiene Franco para extender dichos territorios al socaire de las primeras y fulminantes victorias del Eje (sobre todo con la caída de Francia en junio de 1940), en las que cifra todos sus anhelos y esperanzas. Son, pues, los años en que los sueños imperiales largamente acariciados por los militares africanistas (anexión de la zona francesa del Protectorado marroqui) parece que van a hacerse realidad. Sin embargo, la posición de España en el conflicto (entre la neutralidad y la no beligerancia siempre cercana a Alemania e Italia) y la propia evolución de éste provocan que, a partir de 1945, las ínfulas imperiales y los sueños de expansión territorial se transformen en una política de repliegue para hacer frente al aislamiento internacional. Es entonces cuando la política exterior del régimen busca el acercamiento a los que pueden ser sus principales valedores en el concierto internacional: Hispanoamérica y los países árabes. En este último aspecto, el de la tan cacareada «tradicional amistad hispanoárabe», la presencia española en Marruecos adquiere una nueva relevancia ${ }^{4}$.

En suma, todas estas circunstancias ( $y$ algunas otras que veremos más adelante) permiten aventurar que en estos años las colonias africanas pasan a ocupar un puesto menos secundario en la política española. No obstante, se corre el riesgo de confundir la parte con el todo. Es decir, lo verdaderamente prioritario era, como se deduce de lo expuesto, el Protectorado de Marruecos mientras que el resto de las posesiones africanas merecian una exigua atención. De ahí que tal vez deba hablarse mejor de la política marroquí del régimen de Franco (al menos hasta 1956) y no tanto de una política africana.

Otro aspecto que convendría dilucidar es el del papel jugado en la elaboración y ejecución de una política africana por Carrero Blanco desde la

4 Acerca de la politica exterior del régimen de Franco merece la pena consultarse la síntesis de Espadas Burgos, Manuel (1987). La monografía más reciente y completa sobre la postura de España en la II Guerra Mundial es la de TUSELL, Javier (1995). En lo concerniente a la política hacia lberoamérica existen dos excelentes investigaciones: DEL GADO GOMEZ-ESCALONILLA, Lorenzo (1988); y PARDO SANZ, Rosa (1995). Las relaciones con los paises árabes han sido estudiadas recientemente en la Tesis Doctoral inédita de Algora Weber, María Dolores (1994). V. además PORTERO, Florentino (1989). 
Subsecretaría de la Presidencia del Gobierno. Son muchos los autores que le consideran una pieza clave como responsable directo y máximo de la política colonial y Stanley Payne no duda en afirmar que Carrero dominó la política española en África hasta el momento de su asesinato ${ }^{5}$. Es probable que el archivo de Carrero Blanco, que hasta ahora sólo ha podido ser consultado por el profesor Tusell, nos depare un mayor conocimiento al respecto y despeje las brumas que rodean, al menos en lo concerniente a este punto, a esta figura capital del régimen ${ }^{6}$.

En gran medida, la importancia de Carrero en la política colonial del Franquismo responde a la estratégica posición que ocupaba en Presidencia del Gobierno, órgano del que dependía la administración de las posesiones africanas. La Dirección General de Marruecos y Colonias, que había sido creada por la Dictadura de Primo de Rivera (R. D. de 15 de diciembre de 1925), fue reorganizada en 1931 y suprimida definitivamente en 1934. En septiembre de 1938 el gobierno de Burgos estableció el Servicio Nacional de Marruecos y Colonias, dependiente de Vicepresidencia del Gobierno, que en agosto de 1939, ya con su anterior rango de Dirección General, pasó a incorporarse al Ministerio de Asuntos Exteriores, siendo por entonces ministro el coronel Beigbeder, militar africanista que había sido Alto Comisario en Marruecos desde 1937. Sin embargo, por Ley de 15 de enero de 1942 la Dirección General pasó a depender de Presidencia del Gobierno, coincidiendo significativamente con el hecho de que en la remodelación ministerial de mayo de 1941 Beigbeder hubiese sido sustituido por Serrano Súñer, mucho menos interesado en Marruecos que su antecesor, y Carrero hubiera sido nombrado Subsecretario de la Presidencia. Desde este momento la Dirección General en sus diferentes denominaciones (de Marruecos y Colonias hasta 1956, de Plazas y Provincias Africanas hasta 1968, de Promoción del Sahara hasta 1975 para terminar convirtiéndose en la Comisión Liquidadora de los intereses españoles en el Sahara), y por ende la acción española en África, dependerá única y exclusivamente de Presidencia del Gobierno, es decir, de Carrero Blanco.

Durante los años cuarenta y cincuenta (concretamente entre 1944 y 1968, año en que fallece) la Dirección General estuvo en manos de una figura que todavía hoy es muy poco conocida, José María Díaz de Villegas y Bustamante, general de Estado Mayor, cuya personalidad y gestión merecería una investigación detenida.

PAYNE, Stanley (1987), p. 556.

Sobre Carrero la biografía más completa y solvente con la que contamos es la de TUSELL, J. (1993). 
En estrecha relación con la Dirección General de Marruecos y Colonias se encuentra otro aspecto de capital importancia para entender la vertiente africana del Franquismo: la evolución y características del pensamiento africanista en estos años. Para el profesor Morales Lezcano el africanismo español de posguerra logró constituir una rama guineísta (sic) que convivió con la rama magrebí que contaba con una más larga y rica tradición ${ }^{7}$. Junto a esta revalorización en el terreno ideológico y cultural de los territorios españoles en el África Ecuatorial, en líneas generales el africanismo franquista de estas primeras décadas tuvo un marcado contenido ideológico de carácter esencialista, reivindicativo y nostálgico-propagandístico, según los términos que utiliza el profesor Gonzalo Sanz Casas ${ }^{8}$. Este tipo de discurso paternalista, retórico y propagandístico emanó en la mayoria de los casos de instancias oficiales y persiguió unos fines muy concretos: proporcionar un fundamento teórico e histórico a las reivindicaciones españolas en África, defender la acción de España en sus posesiones, incrementar el conocimiento empírico y la información sobre las mismas con el propósito de favorecer y facilitar la explotación económica de sus recursos, entre otros con menos consecuencias prácticas y mayor contenido retórico.

Probablemente uno de los momentos en los que la exaltación retórica alcanzó grados más notables fue el de los años de la Segunda Guerra Mundial cuando el término Imperio fue profusamente utilizado en la prensa y otros órganos propagandísticos del régimen. Este discurso imperial, cuyas raíces hay que buscarlas tanto en los postulados de la Falange como en el pensamiento autoritario de los hombres de Acción Española, adoptó una doble dirección: "espiritual» y cultural hacia Hispanoamérica (Consejo de la Hispanidad) y político-militar y económica en el caso del Norte de África. Es entonces cuando el pensamiento africanista, como demuestra el significativo libro de Castiella y Areilza (Reivindicaciones de España) y otros no menos sintomáticos de Cordero Torres, adquiere unos tintes más combativos reivindicando sin tapujos una decidida expansión territorial hasta conformar un Imperio español que abarcase todo el Magreb, incluyendo el Oranesado argelino, y las zonas circundantes al Sahara español y Guinea (especialmente Gabón). En contra de lo que algún autor ha afirmado, estas ideas y pretensiones no sólo eran privativas de un grupo de panegiristas exaltados sino que fueron tenidas muy en cuenta por las más altas instancias del régimen como vendría a corroborarlo la ocupación

Mofales lezcano, V (1986), p. 87.

SAnz CASAS, Gonzalo (1993), pp. 356-357. 
de Tánger en junio de 1940, una tentativa que no propició otras operaciones militares similares debido a la propia evolución del conflicto mundial y no a la falta de voluntad de Franco y su gobierno?

Dentro de los centros oficiales del pensamiento africanista sobresale el Instituto de Estudios Africanos (IDEA), creado en junio de 1945, que, por Orden de Presidencia del Gobierno de 10 de julio de 1946, pasó a incorporarse al Consejo Superior de Investigaciones Científicas, afecto al $\mathrm{Pa}$ tronato "Diego Saavedra Fajardo", aunque permaneciendo siempre en íntima conexión y dependencia de la Presidencia del Gobierno a través del Director General de Marruecos y Colonias que, en razón de su cargo, lo era también del Instituto. El IDEA estaba regido por una Junta de Gobierno y constituido por Secciones de Geologia, Geografía, Física, Edafología, Geografía Humana, Etnología, Arqueología y Arte, Botánica, Antropología, Medicina, Farmacognosia, Entomología, Estudios y Derecho. Además de su tarea investigadora, el IDEA llevaba a cabo una labor publicística y divulgadora mediante ciclos de conferencias y publicación de libros, amén de dos revistas cuya consulta es imprescindible para investigar la política y el pensamiento africanista de estos años. El órgano de prensa del Instituto eran los Archivos del Instituto de Estudios Africanos, una revista trimestral que se editó desde junio de 1947 y que llegó a publicar 81 números. La misión de esta revista era la de reflejar la actividad científica del IDEA, mientras que los trabajos de interés general y divulgación para el gran público se reservaban para otra revista: África. Revista de estudios hispano-africanos. Esta publicación, que inició su andadura en 1942, era la continuación de la Revista de tropas coloniales, fundada en Ceuta en 1924 con el general Queipo de Llano como director y que habia dejado de publicarse en julio de 1936 .

Además del IDEA, el Instituto de Estudios Políticos, centro clave en la elaboración del pensamiento franquista de estos años, tenía una Sección de Estudios Africanos, Orientales y Coloniales, dirigida por José Maria Cordero Torres, otra figura enormemente interesante para la reconstrucción del pensamiento africanista e «imperiai», que publicaba desde 1946 unos Cuadernos de Estudios Africanos, centrados en cuestiones de política internacional sobre el estatuto jurídico de las colonias o la génesis de los movimientos nacionalistas en países islámicos y el África subsaharia-

Las principales aspiraciones territoriales se encuentran recogidas en AREILZA, José $M^{a}$ de y CAStiella, Fernando $M^{a}$ (1941) y CORdero TORRES, José $M^{a}(1941 ; 1942)$. Acerca de la ocupación española de Tánger debe consultarse Tusell, J. (1995), pp. 105-123; SuEIro, Susana (1994); Hernando de LarRamendi, Miguel (1988); y Halstead, Charles R. y Carolyn J. (1978). 
na. La presencia de una Sección de estas características en el seno del Instituto de Estudios Políticos revela la importancia que el régimen concedía a todo lo relacionado con África ya que, como ha señalado Stanley Payne, dicho Instituto, concebido como el "brain trust" de la FET, estaba destinado a la formación de los cuadros del Partido y al estudio de toda clase de cuestiones ideológicas y políticas ${ }^{10}$.

En el ámbito del Protectorado marroquí se crearon en los primeros cuarenta el Instituto Muley el Mehdi de estudios marroquíes y el Instituto General Franco para la investigación hispano-árabe, completándose así la política cultural del Franquismo con respecto a las colonias africanas ${ }^{11}$.

Este pensamiento africanista tuvo asimismo un componente economicista destinado a establecer cuál era la riqueza (agrícola, minera, forestal, pesquera, etc.) de las posesiones africanas y si era viable su explotación tanto en términos de eficacia técnica como económica. Con toda seguridad, es éste (el del uso económico que se les deparó a las colonias africanas) el aspecto peor tratado, cualitativa y cuantitativamente, de la presencia española en África, lo que para el periodo que nos ocupa no deja de sorprender si atendemos al hecho de que son los años de la autarquia en los que, dada la búsqueda decidida de la autosuficiencia económica, la reducción drástica de importaciones y la escasa producción agrícola e industrial española, unida a las privaciones y al hambre de la posguerra, resulta en extremo lógico que el régimen se interesase en intensificar al máximo la explotación de los recursos de los territorios africanos con el fin de incrementar las exportaciones y paliar las graves carencias de la población. Para acercarnos, aunque sea someramente, a esta cuestión sólo contamos con un breve y enjundioso estudio de Carlos Velasco, más centrado en elementos de pensamiento y política económico-coloniales que en un estudio pormenorizado y cuantitativo de la realidad económica de las colonias y de su explotación estatal y privada ${ }^{12}$.

A lo largo de la década de 1940 se observa, dentro del campo de la economía y del pensamiento económico español, una potenciación del interés por África como lo demuestran los libros de una serie de autores

10 PAYNE, S. (1985), p. 217

"Sobre el pensamiento africanista del régimen de Franco pueden consultarse MORALES LEZCANO, V. (1986), pp. 83-87; SAEZ DE GOVANTES, Luis (1971), pp. $209-215$ (a título meramente informativo); y DELGADO GOMEZ-ESCALONILLA, L. (1992), pp. 225-231. La única monografia sobre el particular es la de BOSCH, Alfred (1985). Desgraciadamente los archivos del IDEA parecen haber desaparecido o se encuentran dispersos o perdidos según me han testimoniado Lorenzo Delgado y Sisinio Pérez Garzón, investigadores ambos del Centro de Estudios Históricos del CSIC.

12 Velasco Murviedro, Carlos (1988). 
(Perpiñá Grau, Cordero Torres, García Figueras, Fuentes Irurozqui, Cavanna Eguiluz, Vicente Gay, Banciella y Bárcena) y los artículos aparecidos en diversos medios de la época (Nueva Economía Nacional, MUNDO, Información Comercial Española). A grandes rasgos, todos ellos intentan introducir una serie de reformas que mantengan en pie el edificio teórico de la autarquía, cuya práctica no estaba dando los frutos esperados. De esta manera, se empieza a hablar del comercio “limitado" y la autarquía "relativa", para pasar después a la concepción de los Grandes Espacios Económicos y el Espacio Vital de un país y finalizar considerando a las coIonias como el "complemento de la autarquía". Si por un lado España se insertaba en el Gran Espacio Económico euroafricano dirigido por Alemania, cuyos éxitos auguraban un Nuevo Orden económico muy ventajoso para sus aliados aunque en él jugasen el papel de satélites, por otro el Norte de África aparecía como el Espacio Vital por excelencia de la economía española. En los recursos y economías coloniales se buscaba que fuesen primordialmente complementarias de la economía metropolitana bien como fuentes proveedoras de materias primas o bien como nuevos mercados para la colocación de productos industriales. Como escribía en 1940 Pedro Gual Villalbi, hombre representativo de la burguesia industrial catalana y futuro presidente del Consejo de Economía Nacional y ministro sin cartera de Franco (1957-1965), las notas distintivas de las "economías imperialistas" eran las siguientes:

Las premisas económicas del imperialismo completo y perfecto presuponen la independencia del Estado en cuatro aspectos fundamentales: a) en la producción de substancias alimenticias; b) respecto a materias primas y bienes instrumentales indispensables para completar los ciclos productivos; c) respecto a los transportes (principal,... marina mercante) y a los otros instrumentos del cambio; d) en cuanto al área de trabajo, o sea, en punto a las exigencias del ritmo demográfico productivo ${ }^{13}$.

Con este doble objetivo de buscar fuentes de materias primas y mercados se analizaron las economías de los diferentes territorios africanos. Para el caso de Marruecos pronto se comprobó que los recursos que podía ofrecer (minería de hierro, petróleo, productos agrícolas, pesca) no eran tan interesantes y beneficiosos como los que podía demandar, de ahi que se viese en el Protectorado un prometedor mercado para nuestros productos industriales. El enclave Ifni-Sahara era más valorado desde el punto de vista estratégico que económico sin que esto supusiese infrava-

13 Gual Villalbi, Pedro (1940), pp. 595-596. Cit. por Velasco Murviedro, C. (1988), pp. 55-56. 
lorar las posibilidades que ofrecian su riquísimo banco pesquero o sus yacimientos de fosfatos. Guinea, por su parte, cumplía perfectamente el papel de colonia para la obtención de materias primas, especialmente de productos tropicales (café, cacao, maderas, caucho). Así mientras Marruecos ofrecía (por el lado de la demanda) un extenso mercado, deficientemente surtido, a la industria española, Guinea aparecía (desde el lado de la oferta) como una proveedora neta de recursos básicos y de difícil obtención en España. En definitiva, puede afirmarse que los tres enclaves españoles en África ofrecieron a la economía española del momento la posibilidad de completar y superar sus deficiencias gracias a la estructura económica de ellas, de caracteres alternativos entre sí y, a su vez, complementarios de la metropolitana, en los casos de Marruecos y Guinea, sobre todo ${ }^{14}$.

A la hora de llevar a la práctica estos planes económicos y poner en explotación los recursos coloniales tanto la iniciativa privada como la acción del Estado debieron desempeñar un papel importante, compitiendo probablemente en algunos casos entre sí. Dado que en estos años la política industrial del régimen de Franco apostaba por fórmulas autárquicas y de intervencionismo estatal, desplazando en muchas ocasiones al capital privado, cabe dentro de lo posible que el peso de la explotación de nuestras colonias recayese en gran parte sobre el Estado. Por ello sería necesario investigar la vertiente africana del paradigma por antonomasia del proyecto de industrialización autárquico-bélica del primer franquismo: el Instituto Nacional de Industria ${ }^{15}$. El INI fue creado por ley de 25 de septiembre de 1941 y pasó a depender no del Ministerio de Industria, como hubiese sido de esperar, sino de Presidencia del Gobierno. Por tanto, nos encontramos con un caso idéntico al de la Dirección General de Marruecos y Colonias que dependía de Presidencia y no del Ministerio de Asuntos Exteriores. Todo parece apuntar a que tanto la política industrial como la colonial eran de tanta relevancia para el régimen que su gestión debia ser llevada por un órgano administrativo cercano a Franco y en el que Carrero era la pieza clave.

Como demuestran los valiosísimos fondos documentales del archivo del INI, en el seno del Instituto hubo desde el primer momento un serio interés por explotar los recursos de los territorios africanos, interés que se canalizó por medio de varias empresas nacionales. En el Protectorado de

14 Ibidem, p. 88.

15 Sobre el INI puede verse Schwartz, Pedro y Gonzalez, Manuel Jesús (1978) y Martin ACENA, Pablo y COMin, Francisco (1991). 
Marruecos actuaba la Empresa Nacional Torres Quevedo (TQSA), una empresa, como apunta Jordi Catalán, de orientación colonialista y militar, dedicada al mantenimiento y construcción de líneas telefónicas y telegráficas en el Protectorado. Esta empresa, creada en 1943, absorbió en dos años cerca de 32 millones de ptas., un $6 \%$ de las inversiones totales del INI, situándose por su volumen de inversiones sólo por detrás de ENCASO y Elcano, lo que da muestra de su importancia ${ }^{16}$.

Otra empresa, intimamente relacionada con la explotación del banco pesquero canario-sahariano, fue Industrias Pesqueras Africanas S.A. (IPASA) fundada en 1947 por iniciativa de la Empresa Nacional Elcano de construcción naval. En su Consejo de Administración estaban Díaz de Villegas, director general de Marruecos y Colonias, y Nieto Antúnez, futuro ministro de Marina (1962-69) y persona muy cercana al entorno familiar de Franco.

Sin embargo, el sector que contó con una mayor participación del INI fue el de la minería mediante la Empresa Nacional Adaro de investigaciones mineras (1942). Esta empresa encargó distintas investigaciones y prospecciones con la finalidad de establecer las potenciales riquezas del subsuelo africano: el ingeniero de minas José de la Viña estudió las posibilidades petrolíferas del Protectorado a la par que el catedrático Manuel Alía Medina descubría los yacimientos de fosfatos saharianos en 1947. En el período 1948-1956 el INI desarrolló por medio de Adaro los trabajos de evaluación de los yacimientos. En estos años Juan Antonio Suanzes, a la sazón presidente del Instituto y ministro de Industria y Comercio (19451951), realizó un mínimo de tres visitas al Sahara: en enero de 1948, acompañado de los ministros de Agricultura (Rein Segura) y Aire (González Gallarza), al regreso de su viaje oficial a Guinea; en octubre de 1950 en el marco de la vista oficial de Franco al África Occidental Española; y en noviembre de 1951. A pesar de este marcado interés, los fosfatos no sería explotados hasta la década de 1960 primero por parte de la Empresa Nacional Minera del Sahara (ENMINSA) y más tarde de Fosfatos de Bu-Craa (FOSBUCRAA), ambas pertenecientes al INI ${ }^{17}$.

El componente económico de la política africana del régimen de Franco es un tema del que queda aún mucho que investigar pero cuya relevancia queda fuera de toda duda si pretendemos establecer el verdadero alcance de nuestro colonialismo en África. Algunos autores, como Teresa

\footnotetext{
Catalan, Jordi (1995), pp. 233, 236 y 238.
}

Ballestero, Alfonso (1993), pp. 348-349. 
Pereira, han sugerido, pensando fundamentalmente en Guinea, que nuestro colonialismo fue de subsistencia, es decir, que se pretendió que saliese lo más barato posible, invirtiendo poco y tratando de obtener los máximos beneficios posibles con ese bajo nivel de inversiones ${ }^{18}$. El estado actual de las investigaciones no permite, en mi opinión, llegar a conclusiones tan categóricas, pero es una hipótesis de partida válida y sugerente. Además una percepción parecida se encuentra en la tesis que mantiene Alan S. Milward cuando afirma, comparando los diversos resultados del uso económico que España y Portugal hicieron de sus posesiones africanas en los años cincuenta, lo siguiente: los territorios ultramarinos de Portugal contribuyeron en gran medida a la acumulación de reservas en Portugal, permitiendo con ello un descenso en su capacidad para cubrir las importaciones mediante exportaciones(...) Las posesiones españolas en el continente africano, por otro lado, representaban un riesgo para las reservas españolas ${ }^{19}$.

Independientemente de lo arbitraria e infundada que pueda parecernos a priori esta tesis, lo cierto es que nos pone sobre la pista de una perspectiva escasamente utilizada a la hora de enfocar la presencia española en África. Nos referimos, claro está, a una perspectiva comparada que ponga en relación la acción colonial española con la de otros países de nuestro entorno. Del mismo modo que en lo concerniente a la historia de la economía española de los siglo xIx y xx se ha establecido una comparación con el caso italiano (Albert Carreras) o se ha llegado a hablar de un "patrón latino" (Gabriel Tortella) de desarrollo económico que englobaría a España, Portugal e Italia, sería bueno intentar una comparación del modelo colonial español con el de estos dos últimos países en virtud de una serie de semejanzas. Por lo que se refiere al caso de Italia sería interesante comprobar si el modelo colonial implantado por el régimen fascista de Mussolini en Libia y Etiopía pudo ejercer cierta influencia en la política colonial española, lo que no es una suposición descabellada dadas las similitudes doctrinales y de política económica entre ambos regímenes ${ }^{20}$.

No obstante, la comparación más clara y que podría deparar mayores frutos sería con el Imperio portugués (Angola, Mozambique, Guinea-Bissau, Cabo Verde, Santo Tomé y Príncipe). En ambos casos, los territorios africanos actuaron como un mecanismo compensatorio y sustitutorio ante la pérdida de las colonias americanas y, así, Angola y Mozambique pasa-

\footnotetext{
TORRE, Hipólito de la (coord.) (1992), p. 256

MiLwARD, Alan S. (1992), pp. 459-460.

Sobre el colonialismo italiano v. Rochat, Giorgio (1988).
} 
ron a ser considerados el «nuevo Brasil» a la vez que la isla de Fernando Poo se convertía en la imaginación popular en la "nueva Cuba". El uso de trabajo forzado en las plantaciones de cacao por los finqueros de Fernando Poo estaba copiado del sistema de trabajo que se utilizaba en Santo Tomé. Asimismo el sistema de "emancipación" de la población indígena aplicado por las autoridades españolas en Guinea Ecuatorial era muy parecido a la figura del "assimilado" y "nao-civilizado" en las colonias portuguesas. Por otro lado la política de provincialización llevada a cabo por España a finales de la década de 1950 no hacía sino seguir el ejemplo portugués. A esto hay que añadir que, al igual que el régimen de Franco, el Estado Novo de Oliveira Salazar, cuyos presupuestos políticos y económicos eran muy semejantes a los de aquél, desarrolló toda una mística imperial unida a un programa económico cuyo objetivo, como ha indicado el profesor Valentim Alexandre, era el de fazer das colónias um mercado reservado para a produçao da metrópole e um fornecedor de matériasprimas para a indústria portuguesa ${ }^{21}$.

A pesar del carácter general de estas reflexiones, no deberiamos caer en el error de contemplar las colonias españolas en África como un todo homogéneo ya que cada zona poseía ciertas características y condicionantes que la distinguía del resto y que nos obliga a plantear interrogantes diferentes a cada territorio.

El Protectorado de Marruecos era, sin la menor duda, la «joya de la Corona" de nuestro pretendido Imperio africano. Puede decirse que su posesión se medía no tanto en términos de explotación colonial o económica cuanto en virtud de factores militares, de política exterior o de simple prestigio, unidos a ciertos vínculos sentimentales que unian a los generales africanistas, empezando por Franco, a estas tierras. Por la Alta Comisaría española en Marruecos, con sede en Tetuán, pasaron las figuras más prestigiosas del Ejército: Beigbeder (1937-39), Asensio (1939-41), Orgaz (1941-45), Varela (1945-51) y García Valiño (1951-56). Por ello, sería muy interesante estudiar sus políticas y sus relaciones tanto con la Administración jalifiana (el Majzén) como con los generales residentes y las autoridades francesas de la otra zona del Protectorado. Una figura de suma im-

ALEXANDRE, Valentim (1992), p. 40. V. también en ese mismo volumen las aportaciones de TELO, Antonio José (1992) y LOFF, Manuel (1992). Una interpretación global sobre el colonialismo portugués en África puede encontrarse en la sintesis de CLARENCE-SMITH, Gervase (1985). En lo relativo a las relaciones hispanoportuguesas en estos años resulta imprescindible la consulta del reciente libro de JIMENEZ REDONDO, Juan Carlos (1996). La complementariedad de ambas economias, tanto en su vertiente metropolitana como colonial, fue exhaustivamente analizada en un libro de la época: Ruiz Morales, José Miguel (1946). 
portancia en estos años fue la de Tomás García Figueras, secretario general de la Alta Comisaría y asesor sobre las aspiraciones españolas en África de Serrano Súñer en su visita a Berlín en septiembre de 1940, que fue reiteradamente acusado por los ingleses de haber amasado una fortuna aprovechándose de su posición oficial ${ }^{22}$. Otro aspecto que requiere una investigación detallada es el del funcionamiento de las diversas delegaciones de la Alta Comisaría (Asuntos Indígenas, Educación y Cultura, Economía, Industria y Comercio, Obras Públicas, Comunicaciones y Hacienda) con el fin de poder establecer una comparación con las políticas y realizaciones que en el plano económico llevó a cabo Francia en su zona de influencia.

Un caso muy distinto es el de Ifni y el Sahara. Ambos territorios, englobados desde 1946 en la llamada África Occidental Española al mando de un Gobernador General, vivieron siempre en una permanente búsqueda de identidad. Su importancia vino dada en la mayoría de los casos por razones estratégicas (protección y baluarte defensivo de las Islas Canarias) antes que económicas. De esta forma antes de la puesta en explotación de los fosfatos en la década de 1960, su principal activo económico lo constituyó la riqueza de sus bancos pesqueros que en los años cuarenta experimentaron una considerable revalorización como suministradores de productos sustitutivos de bacalao del Norte, una vez que la conflagración mundial había privado a la flota española del acceso a sus caladeros tradicionales y dado que el bacalao era un producto básico en la dieta de los españoles ${ }^{23}$.

Guinea Ecuatorial, por último, fue calificada desde un primer momento (decreto de 11 de julio de 1904) como una colonia de explotación comercial y así fue vista por las autoridades metropolitanas y coloniales a lo largo de su historia como territorio español. Las plantaciones de cacao y café en la isla de Fernando Poo y los recursos madereros de Río Muni justificaron la presencia española en el África Ecuatorial durante esos años. Sin embargo, son aún muchas las cuestiones que aclarar sobre la práctica económica de la colonización de Guinea: el papel (¿monopolístico?) jugado por los poderosos Comités Sindicales (Comité Sindical del Cacao, Sindicato de la Madera, Proguinea del Café) y por el Banco Exterior de España; las veladas acusaciones acerca de la presencia de algunos personajes importantes del régimen como "hacendados imperialistas" (Nieto

2 SUEIRO, S. (1993), p. 303.

23 Sobre este particular v. DIAZ DE LA PAZ, Álvaro (1993) y para una etapa anterior MARTINEZ Mil. LAN, Jesús M. (1992). 
Antúnez, Muñoz Grandes, Díaz de Villegas...), con especial mención a Carrero Blanco como principal accionista de la Compañía Nacional de Colonización Africana (ALENA), creada en Barcelona en 1929 y vinculada al Banco Exterior; la política laboral hacia los trabajadores indígenas, mezcla del modelo portugués y del aplicado en las colonias americanas (comunal y paternalista), el uso del trabajo forzado y los acuerdos y tratados con $\mathrm{Ni}$ geria y Liberia para resolver el suministro regular de mano de obra a la agricultura colonial ${ }^{24}$.

En resumidas cuentas, puede afirmarse sin caer en ningún error o exageración que aún queda mucho por investigar antes de poder ofrecer algo con lo que ya cuentan las historiografías de otros países que tuvieron una presencia multisecular en África: una visión general del colonialismo español en el continente africano; lo que contribuiría a evaluar en sus justos términos lo que supuso la incuestionable dimensión africana del régimen del general Franco.

\section{BIBLIOGRAFIA CITADA}

AleXANDRE, Valentim (1992), «Portugal em África (1825-1974): uma visao geral», en TORRE, H. de la (coord.) (1992), pp. 29-43.

Algora Weber, María Dolores (1994), Las relaciones hispano-árabes durante el período del aislamiento internacional del régimen de Franco (1946-1950); Madrid. Universidad Complu. tense. Tesis Doctoral inédita.

AREILZA, José $M^{a}$ de y CASTIELLA, Fernando $M^{a}$ (1941), Reivindicaciones de España; Madrid, Instituto de Estudios Políticos.

Ballestero, Alfonso (1993), Juan Antonio Suanzes, 1891-1977. La política industrial de la postguerra; León. LID Editorial Empresarial.

BosCH, Alfred (1985), L'Africanisme franquista i I1.D.E.A.(1936-1975); Bellaterra, Universidad Autónoma de Barcelona.

CATALAN, Jordi (1995), La economia española y la segunda guerra mundial; Barcelona, Ariel.

Clarence-Smith. Gervase (1985), The Third Portuguese Empire 1825-1965. A Study in Economic Imperialism; Manchester, Manchester University Press.

Cordero Torres, José $\mathrm{M}^{a}$ (1941), La misión africana de España; Madrid, Ediciones de la Vicesecretaría de Educación Popular.

(1942), Aspectos de la misión universal de España; Madrid, Ediciones de la Vicesecretaría de Educación Popular.

DELGADO GOMEz-ESCALONILLA, Lorenzo (1988), Diplomacia franquista y politica cultural hacia Iberoamérica 1939-1956; Madrid, CSIC.

(1992), Imperio de papel. Acción cultural y politica exterior en el primer franquismo; Madrid, CSIC.

DIAZ DE LA PAZ, Álvaro (1993), "Las pesquerias canario-africanas a la luz de los estudios sobre el banco sahariano (1940-1975)", en MORALES LezCANO, V. (coord.) (1993), pp. 433-441.

Espadas Burgos, Manuel (1987), Franquismo y politica exterior, Madrid, Rialp.

Fusı, Juan Pablo (1985), Franco. Autoritarismo y poder personat; Madrid, EL PAÍS/Aguilar.

$\therefore$ Acerca de estas cuestiones puede verse un planteamiento general en LINIGER-GOUMAZ, Max (1988), especialmente en las pp. 28-45. 
Gual Villalb!, Pedro (1940), Teoría de la política comercial exterior, Barcelona, Editorial Juventud.

HALSTEAD, Charles R. y Carolyn J. (1978), “Aborted Imperialism: Spain's occupation of Tangier, 1940-1945", Iberian Studies, vol. VII, № 2, pp. 53-71.

HeRnando de LaRRAMENDI, Miguel (1988), "Tánger durante la ocupación española, 1940-1945", Actas del Congreso Internacional "El Estrecho de Gibraltar"; Madrid, UNED; t. III. pp. 571 582.

JiMENEZ REDONDO, Juan Carlos (1996), Franco e Salazar. As relaçoes luso-espanhoas no contexto da guerra fría; Lisboa, Assirio \& Alvim.

LINIGER-Goumaz, Max (1988), Small is not always beautiful. The story of Equatorial Guinea; Londres, C. Hurst and Company.

Loff, Manuel (1992), «A propósito de uma negociaçao hispano-portuguesa (1949-54)- o trabalho forçado na base do sistema colonial de produçao nos territórios portugueses de Africa", en TORRE, H. de la (coord.) (1992), pp. 225-235.

MARTin ACENA, Pablo y COMIN, Francisco (1991), INI. 50 años de industrialización en España; Madrid, Espasa Calpe.

Martinez Millan, Jesús M. (1992), Las pesquerias canario-africanas (1800-1914); Las Palmas de Gran Canaria, CIES.

MILWARD, Alan S. (1992), "El sector exterior en la expansión de los años cincuenta: comparación de las exportaciones españolas, italianas y portuguesas", en PRADOS DE LA ESCOSURA, Leandro y ZAMAGNI, Vera (eds.) (1992), pp. 444-461.

Morales Lezcano, Víctor (1986), España y el Norte de África: el Protectorado de España en Marruecos (1912-1956); Madrid, UNED (2 ed.).

(coord.) (1988), I Aula Canarias y el Noroeste de África (1986); Las Palmas de Gran Canaria, Ediciones del Cabildo Insular.

(coord.) (1993), III Aula Canarias y el Noroeste de África (1988); Las Palmas de Gran Canaria, Ediciones del Cabildo Insular.

Pardo Sanz, Rosa (1995), "Con Franco hacia el Imperio". La política exterior española en América Latina, 1939-45; Madrid, UNED.

PAYNe, Stanley (1985), Falange. Historia del fascismo español; Madrid, Sarpe. La $1^{a}$ edición es de 1965 (Paris, Ruedo Ibérico).

(1987), El régimen de Franco, 1936-1975; Madrid, Alianza.

PORTERO, Florentino (1989), Franco, aislado; Madrid, Aguilar.

Prados de la Escosura, Leandro y Zamagni, Vera (eds.) (1992). El desarrollo económico de la Europa del Sur: España e Italia en perspectiva histórica; Madrid, Alianza.

Rochat, Giorgio (1988), I/ colonialismo italiano; Turin, Loescher.

Ruiz Morales, José Miguel (1946), La economía del bloque hispano-portugués; Madrid, Instituto de Estudios Políticos.

SAEz DE Govantes, Luis (1971), El africanismo español; Madrid, Instituto de Estudios AfricanosCSIC

Sanz CASAS, Gonzalo (1993), "Descolonizar la historia de África: el caso de Guinea Ecuatorial" en Morales LezCANo, V. (coord.) (1993), pp. 355-368.

Schwartz, Pedro y González, Manuel Jesús (1978), Una historia del Instituto Nacional de Industria (1941-1976); Madrid, Tecnos.

SUEIRO, Susana (1993), "Sueños de Imperio: las pretensiones territoriales españolas en Marruecos y la diplomacia británica durante la II Guerra Mundial", en TUSELL, J., SUEIRO, S., Marin, J. Ma y Casanova, M. (eds.) (1993), t. II, pp. 299-320.

(1994), «España en Tánger durante la Segunda Guerra Mundial: la consumación de un viejo anhelo", Espacio, tiempo y forma, Serie V. Historia Contemporánea, t. 7, pp. 135-163.

TELO, Antonio José (1992), "Modelos e fases do império português 1890-1961", en TORRE, $H$. de la (coord.) (1992), pp. 69-92.

TORRE, Hipólito de la (coord.) (1992), Portugal, España y África en los últimos cien años: Mérida, UNED-Centro Regional de Extremadura.

TuSELL, Javier (1993), Carrero. La eminencia gris del régimen de Franco; Madrid, Temas de Hoy.

(1995), Franco, España y la II Guerra Mundial; Madrid, Temas de Hoy.

TuSEll, Javier, SuElRo, Susana, MARín, José Ma y CaSanova, Marina (eds.) (1993), El régimen de Franco (1936-1975). Politica y relaciones exteriores; Madrid, UNED. 2 tomos. 
Las colonias españolas en África durante el primer franquismo (1939-1959)...

Velasco Murviedro, Carlos (1988), «Papel económico de las colonias del África noroccidental española en la articulación del Espacio Vital de España (EVE) durante la Autarquía (19361951). El caso de Canarias", en Morales LezCano, V. (coord.) (1988), pp. 49-91. 